\title{
A new life for nitrosocarbonyls in pericyclic reactions
}

\author{
Misal Giuseppe Memeo and Paolo Quadrelli* \\ Department of Chemistry, University of Pavia, Viale Taramelli 12, 27100 Pavia, Italy \\ E-mail: paolo.quadrelli@unipv.it
}

In loving memory of Dr. Achille Piccinini

\begin{abstract}
$\mathrm{Cu}(\mathrm{I} / \mathrm{II})$ salts or Rose Bengal, used in a catalytic amounts, allow for the mild and easy oxidation of hydroxamic acids to nitrosocarbonyls, using air as final oxidant. These new mild oxidative protocols open new vistas in the field of nitroso Diels-Alder (NDA) and ene reactions (ER) of these fugitive intermediates.
\end{abstract}

Keywords: Nitrosocarbonyls, Acyl nitroso compounds, Diels Alder, ene reactions, oxidation, copper catalysis

Nitrosocarbonyls are versatile intermediates widely used in organic synthesis. Their typical reactivity as dienophiles in nitroso Diels-Alder reactions (NDA) as well as super enophiles in ene reactions (ER) has been exploited in the synthesis of relevant biological molecules. ${ }^{1}$ Most of the utility of the NDA reaction in organic syntheses arises from the rich chemistry of the resulting cycloadducts. The 3,6-dihydro-1,2-oxazines, obtained through reaction of nitrosocarbonyls with different dienes, allow for the rapid construction of a wide variety of molecular scaffolds that have been used in the synthesis of natural products, azasugars, amino acid derivatives and carbocyclic analogues. ${ }^{2}$ The acylnitroso ene reaction constitutes a mild and valuable synthetic methodology to obtain allylamine through the direct allylic amination of olefins. Allylamines are versatile and fundamental building blocks for the construction of $\alpha$ - and $\beta$-amino acids, alkaloids and carbohydrate derivatives. ${ }^{2,3}$

Despite the widespread application of these fleeting intermediates, the chemistry of nitrosocarbonyls remains somewhat underdeveloped because of the short lifetime of these fugitive transient species. ${ }^{2}$ Due to the high reactivity of nitrosocarbonyls, they are usually generated in situ through the oxidation of hydroxamic acids. Since their discovery by Kirby ${ }^{4,5}$ and Keck, ${ }^{6}$ most of the generation methods rely upon oxidation protocols mediated by stoichiometric amounts of oxidant such as sodium periodate, iodosobenzene diacetate or, more recently $\mathrm{Ru}(\mathrm{II}), \mathrm{Ir}(\mathrm{I}), \mathrm{Fe}(\mathrm{III}), \mathrm{Cu}(\mathrm{I})$ or peroxides. ${ }^{7}$ 


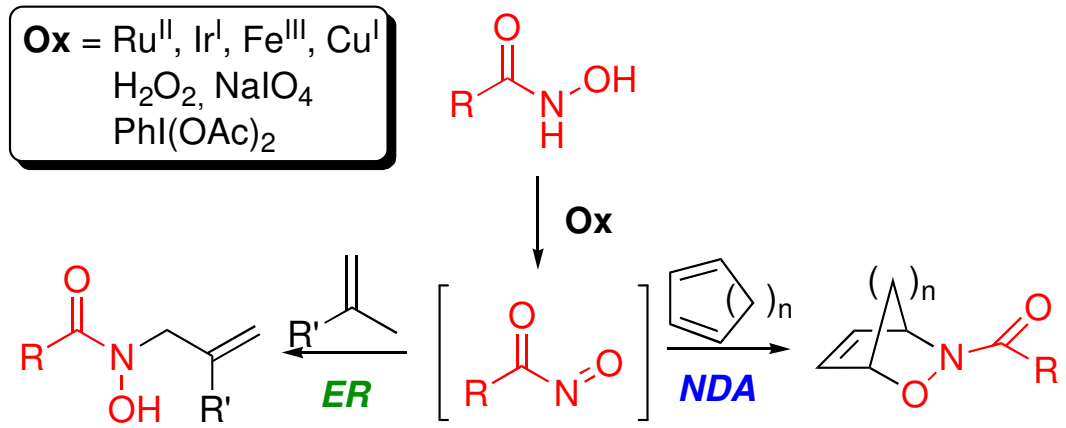

Scheme 1. Oxidative generation and trapping of nitrosocarbonyls by NDA or ER.

However most of the cited methods have certain limitions. ${ }^{8}$ The use of stoichiometric amounts of oxidant often lead to poor atom economy, and some of these oxidants are particularly expensive and environmentally hazardous. ${ }^{9}$ Further, strong oxidative conditions limit the use of functional groups and can reduce the stability of the formed adducts. ${ }^{2}$ Moreover, an excess of olefin is required in ER in order to obtain high yields of ene product. ${ }^{10,11}$

In the last two years attention has been paid to developing new generation methods compatible with a wider plethora of functional groups, and more environmentally friendly. In 2011, Whiting and Read de Alaniz independently demonstrated that the use of copper(II) salts in aerobic conditions as catalytic system is able to readily generate nitrosocarbonyl derivatives starting from the corresponding $N$-hydroxycarbamates. Starting from the NDA reaction, Whiting and coworkers ${ }^{12}$ pointed out how $\mathrm{CuCl}_{2}$, in the presence of a 2-ethyl-2-oxazoline as a ligand and oxygen, is an effective catalyst for the room temperature oxidation of hydroxamic acids to acylnitroso derivatives, which are efficiently trapped in situ via both inter- and intramolecular hetero-Diels-Alder reactions with several dienes (Scheme 2). ${ }^{12}$

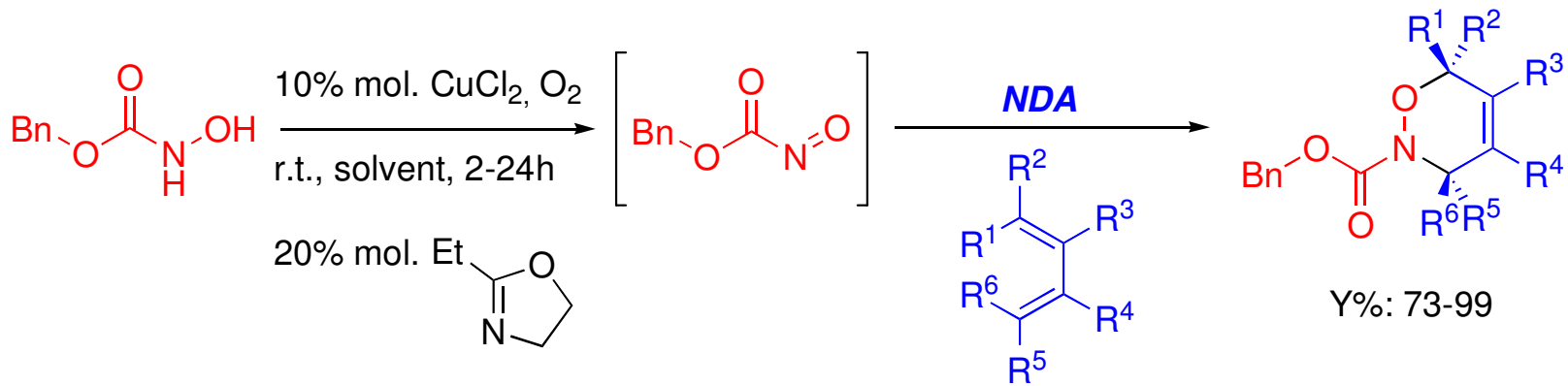

Scheme 2. NDA between in situ generated nitrosocarbonyls and different dienes.

Read de Alaniz and co-workers ${ }^{13}$ showed that is possible to avoid the use of pure oxygen using copper(I) chloride (20 mol\%) and pyridine (5 mol\%). Under these conditions the amount of oxygen present in the atmosphere is sufficient to promote the clean conversion of different $N$ substituted hydroxylamines into the corresponding oxazines. In the reaction scope the elevated 
range of application is remarkable. Acylnitroso, nitrosoformate, nitrosoformamide, as well as iminonitroso, arylnitroso and $P$-nitrosophosphine oxide derivatives, in situ generated, have been made to react with different dienes always yielding the desired products in very good yields (Figure 1).<smiles>[R]C(=O)N1OC2C=CC1C2</smiles>

Y\%: 58-99<smiles>[R]C(=O)N1OC2C=CC1C2</smiles>

Y\%: 94<smiles>[R]C(=O)N1OC([R])C([R])=C([R8])C1[R7]</smiles>

Y\%: 43-80

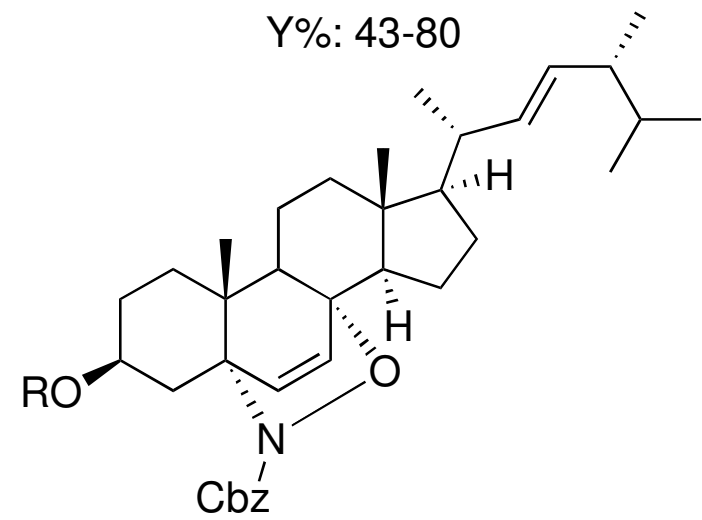

Y\%: 86-99

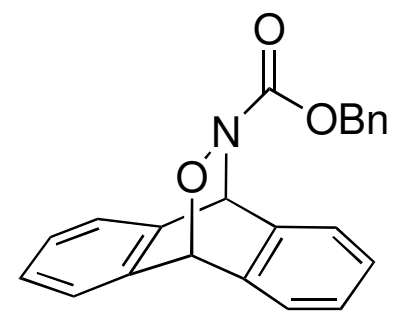

Y\%: 99

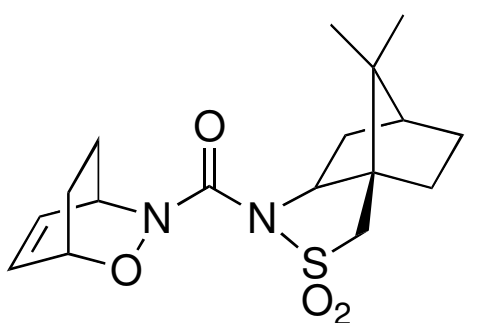

Y\%: 99 $>20: 1 \mathrm{dr}$

Figure 1. Examples of NDA products obtained using $\mathrm{CuCl}$, pyridine and air.

The group from Santa Barbara applied the same procedure used for the NDA reaction to the ene reaction of $N$-hydroxycarbamate bearing a tetrasubstituted olefin. Using this intramolecular ER as reference, they found the proper conditions to extend the reaction scope to more than 20 different substrates, performing intra- or intermolecular ene reactions in good yields. $^{14}$

At the beginning of this year, Tan and co-workers, ${ }^{15}$ in Singapore, encouraged by the aforementioned results, became interested in developing an alternative metal-free approach to generate the acylnitroso species by means of light. Rose Bengal, an inexpensive and readily available organic dye, was demonstrated to be a photoredox catalyst for the formation of transient nitrosocarbonyls under visible light irradiation (Scheme 3). ${ }^{15}$ This photoreaction takes place through an electron transfer process between the Rose Bengal and the hydroxamic acids. The process somewhat represents an alternative to the ER via singlet oxygen of alkenes under photosensitization with Rose Bengal with visible light. Moreover, it is an environmentally friendly allylic amination methodology that avoids the use of a metal catalyst and stoichiometric amounts of oxidant, is operationally simple, and uses air as the terminal oxidant. 
In conclusion, in this article we highlight new mild reaction conditions for the in situ aerobic generation of acylnitroso species. Air is the ultimate oxidant, whether using an organic dye such as Rose Bengal, or by employing copper salts. These methods are operationally simple and represent so far the mildest and more environmentally sustainable procedure to generate nitrosocarbonyls. A wide range of NDA and ER have been explored, affording the desired products from moderate to quantitative yields. New advances can be predicted in organic synthesis for these fugitive intermediates exploiting the procedures described in this paper.

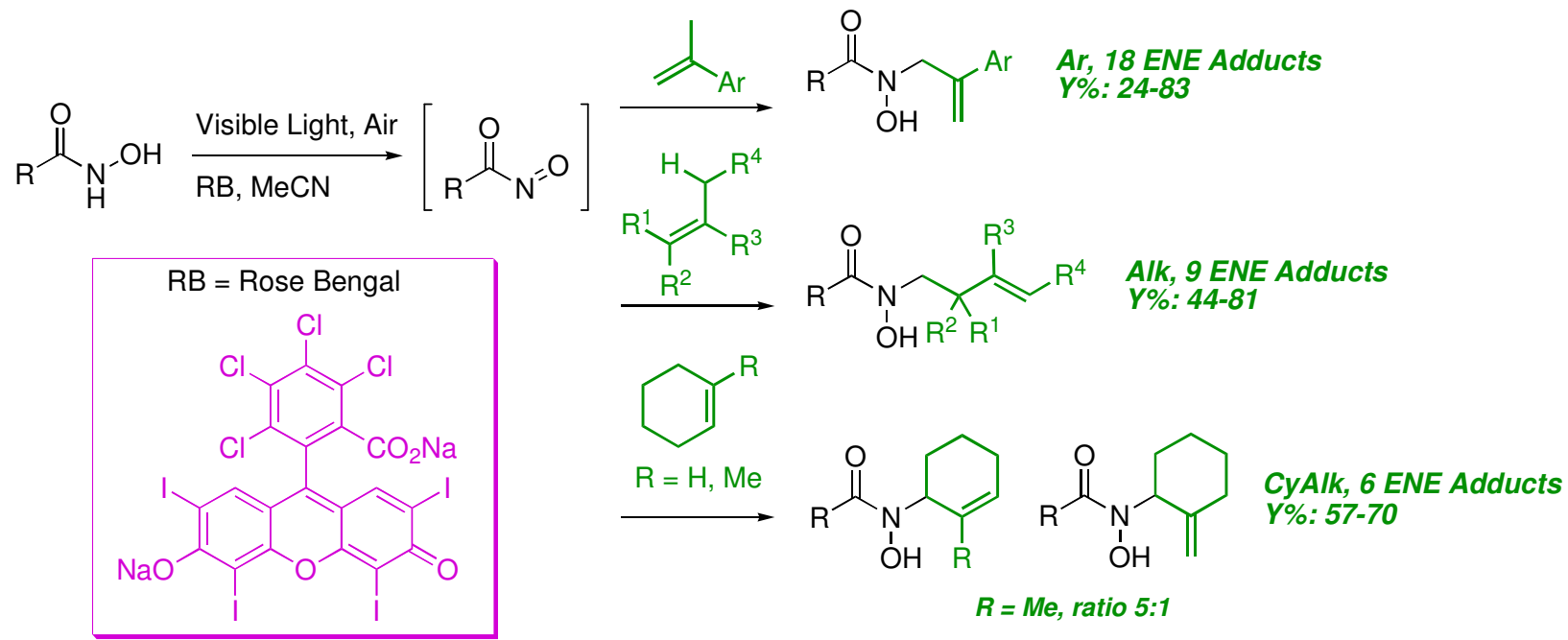

Scheme 3. Photocatalytic approach for the ER.

\section{Acknowledgements}

We acknowledge financial support from the University of Pavia and MIUR (PRIN 2008, CUP: F11J10000010001 and PRIN 2011, CUP B11J12002450001).

\section{References}

1. Bodnar, B. S.; Miller, M. J. Angew. Chem. Int. Ed. 2011, 50, 5630.

http://dx.doi.org/10.1002/anie.201005764

2. Adam, W.; Krebs, O. Chem. Rev. 2003, 103, 4131.

http://dx.doi.org/10.1021/cr030004x

3. Iwasa, S.; Fakhruddin, A.; Nishiyama, H. Mini-Rev. Org. Chem. 2005, 2, 157. http://dx.doi.org/10.2174/1570193053544445

4. Kirby, G. W. Chem. Soc. Rev. 1977, 6, 1. http://dx.doi.org/10.1039/cs9770600001 
5. Kirby, G. W.; McGuian, H.; McLean, D. J. Chem. Soc. Perkin Trans. 1 1985, 1961.

6. Keck, G. E.; Webb, R. R.; Yates, J. B. Tetrahedron 1981, 37, 4007. http://dx.doi.org/10.1016/S0040-4020(01)93275-2

7. Fakhruddin, A.; Iwasa, S.; Nishiyama, H.; Tsutsumi, K. Tetrahedron Lett. 2004, 45, 9323. http://dx.doi.org/10.1016/j.tetlet.2004.10.128

8. Atkinson, D.; Kabeshov, M. A.; Edgar, M.; Malkov, A. V. Adv. Synth. Catal. 2011, 353, 3347.

http://dx.doi.org/10.1002/adsc.201100632

9. Sheldon, R. A.; Arends, I. W. C. E.; Hanefeld, U. Green Chemistry and Catalysis, WileyVCH, Eds.; Weinheim 2007, pp. 1-47.

10. Adam, W.; Bottke, N.; Krebs, O.; Saha-Moller, C. R. Eur. J. Org. Chem. 1999, 1963.

11. Kalita, B.; Nicholas, K. M. Tetrahedron Lett. 2005, 46, 1451. http://dx.doi.org/10.1016/j.tetlet.2005.01.024

12. Chaiyaveij, D.; Cleary, L.; Batsanov, A. S.; Marder, T. B.; Shea, K. J.; Whiting, A. Org. Lett. 2011, 13, 3442.

http://dx.doi.org/10.1021/o1201188d

13. Frazier, C. P.; Bugarin, A.; Engelking, J. R.; Read de Alaniz, J. Org. Lett. 2012, 14, 3620. http://dx.doi.org/10.1021/ol301414k

14. Frazier, C. P.; Engelking, J. R.; Read de Alaniz, J. J. Am. Chem. Soc. 2011, 133, 10430. http://dx.doi.org/10.1021/ja204603u

15. Teo, Y. C.; Pan, Y.; Tan, C. H. Chem. Cat. Chem. 2013, 5, 235.

\section{Authors' Biographies}

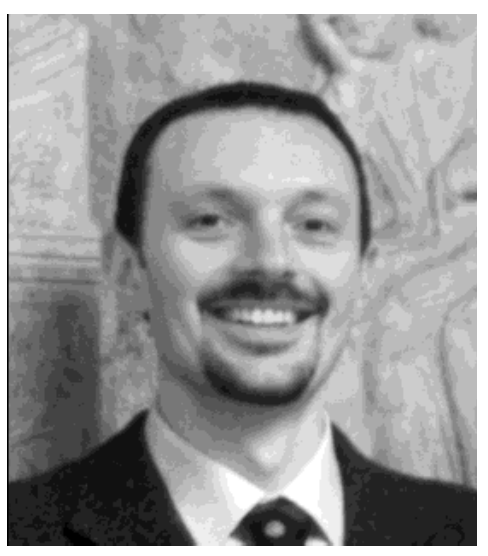

Paolo Quadrelli was born in 1961. He received his degree in chemistry in 1986 at the University of Pavia. In 1990 he obtained the PhD degree at the same University under the supervision of G. Desimoni. Then he moved to the R\&D Lab. of ENI group until 1992, when he returned as Researcher in the P. Caramella group. In 1996 he joined the group of R. Grigg at the University of Leeds. He is currently associate professor of Organic Chemistry at the Department 
of Chemistry of the University of Pavia. His research interests are pericyclic reactions, chemistry of 1,3-dipoles, transition metal-catalyzed reactions and solid phase chemistry.

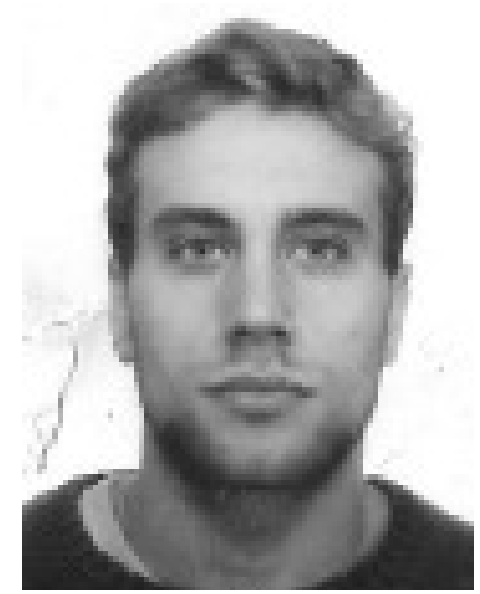

Misal Giuseppe Memeo was born near Milan in 1986. On 2010 he received his degree in chemistry at the University of Pavia. He is currently working on his $\mathrm{PhD}$ under the guidance of P. Quadrelli at the same University. He was visiting PhD student in D. Spring Group at the University of Cambridge. His current researches focus on the synthesis of nucleoside analogues and peptidomimetics. 